\title{
Correction to: Elemental mapping of some collected gold samples from Al-Amar gold mine in Saudi Arabia
}

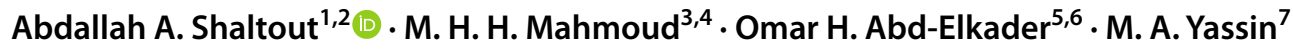

Published online: 21 January 2021

c) Springer-Verlag GmbH Germany, part of Springer Nature 2021

\section{Correction to: Environmental Earth Sciences (2020) 79:527 https://doi.org/10.1007/s12665-020-09271-1}

In the original publication one of the author name was published wrongly unfortunately. The correct author name should be "M. H. H. Mahmoud" instead of "Mohamed H. Mahamoud".

Publisher's Note Springer Nature remains neutral with regard to jurisdictional claims in published maps and institutional affiliations.

The original article can be found online at https://doi.org/10.1007/ s12665-020-09271-1.

Abdallah A. Shaltout

aa.shaltout@nrc.sci.eg

M. H. H. Mahmoud

mheshamm@gmail.com

1 Physics Department, Faculty of Science, Taif University, P. O. Box 11099, Taif 21944, Saudi Arabia

2 Spectroscopy Department, Physics Division, National Research Centre, El Behooth St., Dokki, Cairo 12622, Egypt

3 Chemistry Department, Faculty of Science, Taif University, P. O. Box 11099, Taif 21944, Saudi Arabia

4 Central of Metallurgical R \& D Institute, P. O. Box 87, Helwan, Cairo, Egypt

5 Physics \& Astronomy Department, Science College, King Saud University, P.O. Box 2455, Riyadh 11451, Saudi Arabia

6 Physics Division, Electron Microscope \& Thin Films Department, National Research Centre, El Behooth St., Giza 12622, Egypt

7 Botany \& Microbiology Department, Science College, King Saud University, P.O. Box 2455, Riyadh 11451, Saudi Arabia 